\title{
Four-Node Generalized Conforming Membrane Elements with Drilling DOFs Using Quadrilateral Area Coordinate Methods
}

\author{
Xiao-Ming Chen, ${ }^{1}$ Song Cen, ${ }^{2,3,4}$ Jian-Yun Sun, ${ }^{1}$ and Yun-Gui Li ${ }^{1}$ \\ ${ }^{1}$ China State Construction Technical Center, Beijing 101300, China \\ ${ }^{2}$ Department of Engineering Mechanics, School of Aerospace Engineering, Tsinghua University, Beijing 100084, China \\ ${ }^{3}$ High Performance Computing Center, School of Aerospace Engineering, Tsinghua University, Beijing 100084, China \\ ${ }^{4}$ Key Laboratory of Applied Mechanics, School of Aerospace Engineering, Tsinghua University, Beijing 100084, China
}

Correspondence should be addressed to Song Cen; censong@tsinghua.edu.cn

Received 26 August 2014; Accepted 1 September 2014

Academic Editor: Zhiqiang Hu

Copyright (C) 2015 Xiao-Ming Chen et al. This is an open access article distributed under the Creative Commons Attribution License, which permits unrestricted use, distribution, and reproduction in any medium, provided the original work is properly cited.

Two 4-node generalized conforming quadrilateral membrane elements with drilling DOF, named QAC4 $\theta$ and QAC4 $\theta \mathrm{M}$, were successfully developed. Two kinds of quadrilateral area coordinates are used together in the assumed displacement fields of the new elements, so that the related formulations are quite straightforward and will keep the order of the Cartesian coordinates unchangeable while the mesh is distorted. The drilling DOF is defined as the additional rigid rotation at the element nodes to avoid improper constraint. Both elements can pass the strict patch test and exhibit better performance than other similar models. In particular, they are both free of trapezoidal locking in MacNeal's beam test and insensitive to various mesh distortions.

\section{Introduction}

It is well known that adding the drilling degree of freedom (DOF) at each node of a plane membrane element can enhance the element performance without increasing the number of the element nodes. Furthermore, a plane membrane element with drilling DOFs can be combined with a plate bending element to form a flat-shell element, which can avoid the problem of singular coefficients associated with the DOFs in the direction normal to the plane of the shell element.

The research on drilling DOF can be traced to the 1960s. Olson and Bearden [1] proposed the first valuable triangular membrane element with drilling DOF. However, since the rotations of two adjacent edges are assumed to be equal, this element may not converge to the correct solution. Another model, a hybrid displacement triangular element with drilling DOF, was then proposed by Mohr [2], but its variational theory is not sufficient. The definition of the drilling DOFs proposed by Allman [3,4] can be treated as a milestone for this topic, in which a quadratic displacement approximation was introduced to supplement the drilling
DOFs at element nodes. Based on Allman's work, numerous researches on plane elements with drilling degrees of freedom have been accomplished, such as the models proposed by Bergan and Felippa [5], Cook [6, 7], MacNeal and Harder [8], Yunus et al. [9], Hughes and Brezzi [10], Ibrahimbegovic et al. [11], Iura and Atluri [12], Cazzani and Atluri [13], Piltner and Taylor [14], Geyer and Groenwold [15], Pimpinelli [16], Groenwold et al. [17], Choi et al. [18], Choo et al. [19], Zhang and Kuang [20], Kugler et al. [21], and Cen et al. [22]. Long et al. [23-25] presented a new definition on the drilling DOFs. They treated these DOFs as the additional rigid rotations at the element nodes, so that the change of the angle between two adjacent edges along with the element deformation is allowed, and the rotation of the element edge has definite relation with the nodal drilling DOFs. Based on this assumption, the triangular element GT9 series and the quadrilateral element GQ12 series were developed. These elements all exhibit good performance [26], and among these models the quadrilateral element GQ12M8 is the best one.

It is also well known that most quadrilateral elements use the isoparametric coordinates $(\xi, \eta)$ to express their formu- 
lations. Lee and Bathe [27] have studied the influence of mesh distortions on the isoparametric membrane elements and showed that the serendipity family is quite sensitive to the mesh distortions. They concluded that the nonlinear transformation between the isoparametric (local) and the Cartesian (global) coordinates leads to such problem. Although the assumed displacement fields may contain highorder terms of $\xi$ and $\eta$, their complete order in Cartesian coordinates $x$ and $y$ will degrade significantly once the meshes are distorted, which will lead to low accuracy. In order to make the isoparametric displacement fields satisfy second order completeness in Cartesian coordinates, even fourth order isoparametric terms should be introduced, such as the abovementioned element GQ12M8. This makes the element formulations quite complicated.

For overcoming this inherent defect of the isoparametric coordinates, Long et al. [28, 29] developed the first kind of quadrilateral area coordinate method QACM-I. The QACMI possesses an important character: the transformation between the area and the Cartesian coordinate systems is always linear. Thus, the disadvantage of the isoparametric coordinate system is avoided from the outset. Then, this new natural coordinate system was successfully applied to develop new finite element models. Soh et al. [30] constructed two 8-node plane quadrilateral generalized conforming elements which are insensitive to mesh distortion. Chen et al. [31] proposed two 4-node quadrilateral membrane elements AGQ6-I and AGQ6-II, which exhibit excellent performance in high-order benchmark examples; particularly, both can perfectly pass MacNeal's thin beam test [32]. These two 4node elements arouse the interests in further studies on the QACM-I. Cen et al. [33] derived out the analytical element stiffness matrix of AGQ6-I and developed a family of the quadrilateral plane membrane elements [34]. Du and Cen [35] extended the element AGQ6-I to geometrically nonlinear analysis. Cardoso et al. [36-38] introduced the element AGQ6-I to develop distortion-immune shell elements for linear, nonlinear, and dynamic fracture analyses. Wang and Sun [39] used the element AGQ6-II to formulate a new corotational nonlinear shell element. Chen et al. [40] modified the element AGQ6-I to make it pass the strict patch test. Li [41] improved the formulations and generalized them to simulate coupled solid-deformation/fluid-flow for porous geomaterials. Cardoso and Yoon [42], Prathap and Senthilkumar [43], and Flajs et al. [44] discussed the convergence for related AGQ6 models. Besides the above plane elements, the QACMI has also been successfully employed to develop thin plate [45], Mindlin-Reissner plate [46], laminated composite plate [47], and shell models [48-51].

Since the QACM-I contains four area coordinate components $\left(L_{1}, L_{2}, L_{3}\right.$, and $\left.L_{4}\right)$, among which only two are independent, users may be confused on how to formulate a complete high-order polynomial. In view of this disadvantage, Chen et al. [52] proposed the second kind of quadrilateral coordinate method QACM-II. This QACM-II uses two midlines of opposite sides as the coordinate axes and defines only two independent coordinate components $Z_{1}$ and $Z_{2}$. The element formulations expressed by the QACM-II are quite simpler than those in terms of the QACM-I $[52,53]$. In 2010,

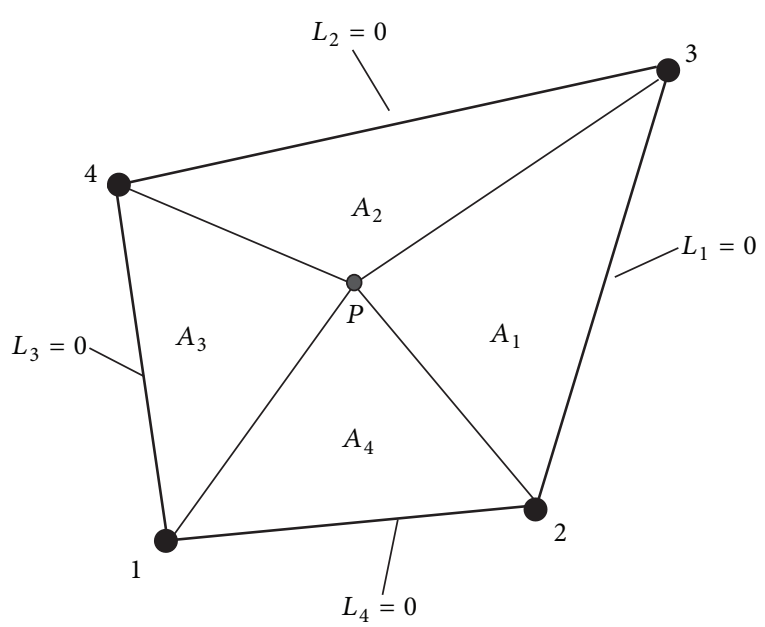

FIGURE 1: Definition of the quadrilateral area coordinates $L_{i}$ of the QACM-I.

Long et al. [54] established the third kind of quadrilateral area coordinate method QACM-III. It takes two diagonals as the zero coordinate axes to define the two independent coordinate components $T_{1}$ and $T_{2}$. All the three kinds of area quadrilateral coordinate can be used simultaneously in one element, which will make the formulations quite simple and straightforward.

In this paper, by combination with the definition of drilling DOFs proposed by Long et al. [23-25], a new plane membrane element with drilling DOFs, denoted by QAC4 $\theta$, was firstly developed by using the QACM-III. Then, by introducing a generalized bubble displacement field in terms of QACM-II into the element QAC4 4 , a more accurate and robust element, denoted by QAC $4 \theta \mathrm{M}$, was constructed. Both elements can pass the strict patch test and exhibit better performance than other similar models. It is demonstrated again that the quadrilateral area coordinate methods are effective tools for developing high-performance quadrilateral finite element models.

\section{Brief Reviews on the Quadrilateral Area Coordinate Methods}

2.1. QACM-I [28, 29]. As shown in Figure 1, the position of an arbitrary point $P$ within a quadrilateral element $\overline{1234}$ is specified by the area coordinates $L_{1}, L_{2}, L_{3}$, and $L_{4}$, which are defined as

$$
L_{i}=\frac{A_{i}}{A}, \quad(i=1,2,3,4),
$$

where $A$ is the area of the quadrilateral element; $A_{i}(i=$ $1,2,3,4)$ are the areas of the four triangles constructed by point $P$ and four element sides $\overline{23}, \overline{34}, \overline{41}$, and $\overline{12}$, respectively. $L_{1}, L_{2}, L_{3}$, and $L_{4}$ can be expressed in terms of Cartesian coordinates $(x, y)$ as follows:

$$
L_{i}=\frac{1}{2 A}\left(a_{i}+b_{i} x+c_{i} y\right), \quad(i=1,2,3,4)
$$


with

$$
\begin{array}{r}
a_{i}=x_{j} y_{k}-x_{k} y_{j}, \quad b_{i}=y_{j}-y_{k}, \quad c_{i}=x_{k}-x_{i}, \\
(i=1,2,3,4 ; j=2,3,4,1 ; k=3,4,1,2) .
\end{array}
$$

Four dimensionless shape parameters $g_{1}, g_{2}, g_{3}$, and $g_{4}$ to each of the quadrangles, as shown in Figure 2, must be defined as

$$
\begin{array}{r}
g_{1}=\frac{A^{\prime}}{A}, \quad g_{2}=\frac{A^{\prime \prime}}{A}, \quad g_{3}=1-g_{1}, \quad g_{4}=1-g_{2}, \\
\left(0 \leq g_{i} \leq 1\right),
\end{array}
$$

where $A^{\prime}$ and $A^{\prime \prime}$ are the areas of $\Delta 124$ and $\Delta 123$, respectively. Different values of these shape parameters mean different shapes of a quadrangle. And the area coordinates of four corner nodes can be obtained:

$$
\begin{array}{ll}
\text { Node } 1 & \left(g_{2}, g_{4}, 0,0\right) \\
\text { Node } 2 & \left(0, g_{3}, g_{1}, 0\right) \\
\text { Node } 3 & \left(0,0, g_{4}, g_{2}\right) \\
\text { Node } 4 & \left(g_{3}, 0,0, g_{1}\right) .
\end{array}
$$

The relations between the area coordinates $L_{i}$ and the isoparametric coordinates $(\xi, \eta)$ are

$$
\begin{aligned}
L_{1} & =\frac{1}{4}(1-\xi)\left[g_{2}(1-\eta)+g_{3}(1+\eta)\right] \\
L_{2} & =\frac{1}{4}(1-\eta)\left[g_{4}(1-\xi)+g_{3}(1+\xi)\right] \\
L_{3} & =\frac{1}{4}(1+\xi)\left[g_{1}(1-\eta)+g_{4}(1+\eta)\right] \\
L_{4} & =\frac{1}{4}(1+\eta)\left[g_{1}(1-\xi)+g_{2}(1+\xi)\right] .
\end{aligned}
$$

2.2. QACM-II [52]. As shown in Figure $3, M_{i}(i=1,2,3,4)$ are the midside points of element sides $\overline{23}, \overline{34}, \overline{41}$, and $\overline{12}$, respectively. Thus, the position of an arbitrary point $P$ within the quadrilateral element $\overline{1234}$ can be uniquely specified by the two-component area coordinates $Z_{1}$ and $Z_{2}$ (QACM-II), which are defined as

$$
Z_{1}=4 \frac{\Omega_{1}}{A}, \quad Z_{2}=4 \frac{\Omega_{2}}{A},
$$

where $\Omega_{1}$ and $\Omega_{2}$ are the generalized areas of $\triangle P M_{2} M_{4}$ and $\triangle P M_{3} M_{1}$, respectively. It must be noted here that the values of generalized areas $\Omega_{1}$ and $\Omega_{2}$ can be both positive and negative: for $\triangle P M_{2} M_{4}$ (or $\triangle P M_{3} M_{1}$ ), if the permutation order of points $P, M_{2}$, and $M_{4}$ (or $P, M_{3}$, and $M_{1}$ ) is anticlockwise, a positive $\Omega_{1}$ (or $\Omega_{2}$ ) should be taken; otherwise, $\Omega_{1}$ (or $\Omega_{2}$ ) should be negative.
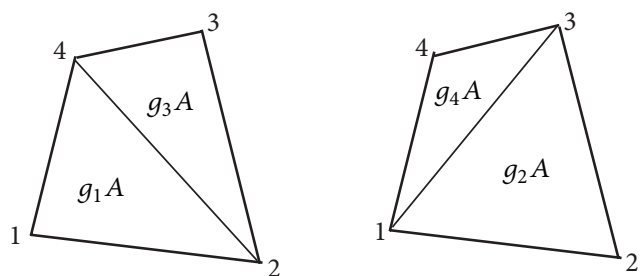

Figure 2: Definitions of $g_{1}, g_{2}, g_{3}$, and $g_{4}$.

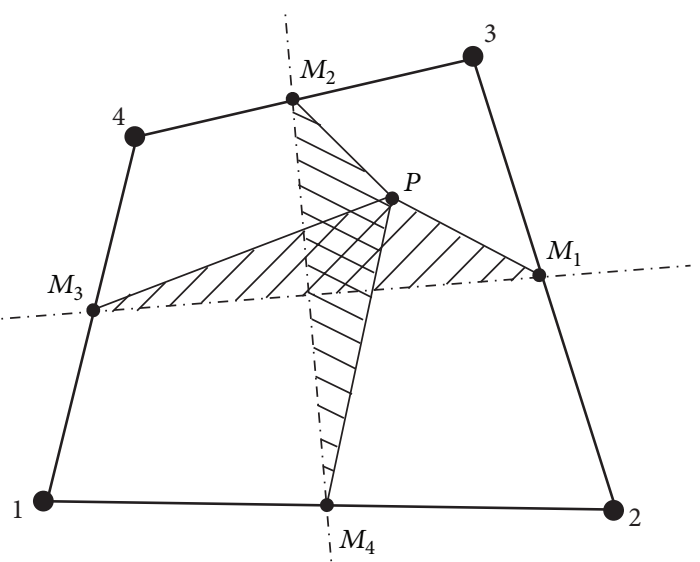

Figure 3: Definition of the quadrilateral area coordinates $Z_{i}$ of QACM-II.

Thus, the local coordinates of the corner nodes and midside points can be obtained:

$$
\begin{aligned}
& \text { Node } 1 \quad\left(-g_{1}-g_{2},-g_{1}-g_{4}\right) \\
& \text { Node } 2\left(g_{1}+g_{2},-g_{2}-g_{3}\right) \\
& \text { Node } 3\left(g_{3}+g_{4}, g_{2}+g_{3}\right) \\
& \text { Node } 4 \quad\left(-g_{3}-g_{4}, g_{1}+g_{4}\right) \\
& M_{1} \quad(1,0) \quad M_{2} \quad(0,1) \\
& M_{3} \quad(-1,0) \quad M_{4} \quad(0,-1) \text {. }
\end{aligned}
$$

The relations between the QACM-II and the QACM-I are

$$
\begin{array}{r}
Z_{1}=\frac{1}{A}\left[\left(a_{3}-a_{1}\right)+\left(b_{3}-b_{1}\right) x+\left(c_{3}-c_{1}\right) y\right]+\bar{g}_{1} \\
Z_{2}=\frac{1}{A}\left[\left(a_{4}-a_{2}\right)+\left(b_{4}-b_{2}\right) x+\left(c_{4}-c_{2}\right) y\right]+\bar{g}_{2} \\
\text { with } \bar{g}_{1}=g_{2}-g_{1}, \quad \bar{g}_{2}=g_{3}-g_{2} .
\end{array}
$$

And $Z_{1}$ and $Z_{2}$ can also be expressed in terms of $\xi$ and $\eta$ as follows:

$$
\begin{aligned}
& Z_{1}=\xi+\bar{g}_{2} \xi \eta \\
& Z_{2}=\eta+\bar{g}_{1} \xi \eta .
\end{aligned}
$$

It can be seen that the new area coordinates $Z_{1}$ and $Z_{2}$ will degenerate to the isoparametric coordinates $\xi$ and $\eta$ for rectangular element cases. 

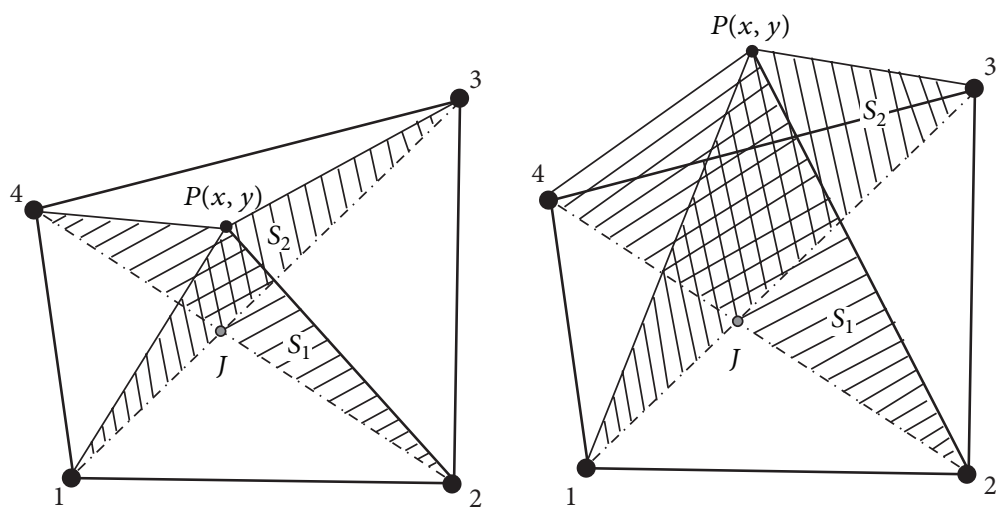

FIgURE 4: Definition of the quadrilateral area coordinates $T_{i}$ of the QACM-III.

2.3. QACM-III [54]. As shown in Figure $4, \overline{13}$ and $\overline{24}$ are the two diagonals of the quadrilateral $\overline{1234}$. Then, the position of an arbitrary point $P$ within or outside the quadrilateral $\overline{1234}$ can be uniquely specified by the two-component area coordinates $T_{1}$ and $T_{2}$ (QACM-III), which are defined as

$$
T_{1}=\frac{S_{1}}{A}, \quad T_{2}=\frac{S_{2}}{A},
$$

where $S_{1}$ and $S_{2}$ are the generalized areas of $\triangle P 42$ and $\triangle P 13$, respectively. The values of generalized areas $S_{1}$ and $S_{2}$ can be both positive and negative: for $\triangle P 42$ (or $\triangle P 13$ ), if the permutation order of the points $P, 4$, and 2 (or $P, 1$, and 3 ) is anticlockwise, a positive $S_{1}$ (or $S_{2}$ ) should be taken; otherwise, $S_{1}\left(\right.$ or $\left.S_{2}\right)$ should be negative.

Then, the local coordinates of the corner nodes can be written as

$$
\begin{aligned}
& \text { node } 1 \quad\left(-g_{1}, 0\right) \quad \text { node } 2 \quad\left(0,-g_{2}\right) \\
& \text { node } 3\left(g_{3}, 0\right) \text { node } 4\left(0, g_{4}\right) \text {. }
\end{aligned}
$$

The relations between the QACM-III and the QACM-I are

$$
\begin{aligned}
& T_{1}=g_{3}-L_{1}-L_{2}=L_{3}+L_{4}-g_{1} \\
& T_{2}=g_{4}-L_{2}-L_{3}=L_{4}+L_{1}-g_{2} .
\end{aligned}
$$

And $T_{1}$ and $T_{2}$ can also be expressed in terms of $\xi$ and $\eta$ as follows:

$$
\begin{gathered}
T_{1}=\frac{1}{4}\left[\xi+\eta+\left(g_{3}-g_{1}\right)(1+\xi \eta)\right] \\
T_{2}=\frac{1}{4}\left[-\xi+\eta+\left(g_{4}-g_{2}\right)(1-\xi \eta)\right] .
\end{gathered}
$$

\section{Definition of the Drilling DOFs}

As shown in Figure 5, Long et al. [23-25] defined the drilling DOFs $\theta_{i}$ as the additional rigid rotations at the element nodes.

The characteristics of this definition are as follows.

(1) The change of the angle between two adjacent sides along with the element deformation is allowed.
(2) The rotation $\theta$ of the element side has definite relation with the nodal drilling freedom $\theta_{i}$.

In this definition, the displacement fields within the domain of an element are assumed to include two parts:

$$
\mathbf{u}=\mathbf{u}^{0}+\mathbf{u}^{\theta}=\left\{\begin{array}{l}
u^{0} \\
v^{0}
\end{array}\right\}+\left\{\begin{array}{l}
u^{\theta} \\
v^{\theta}
\end{array}\right\}
$$

where $\mathbf{u}^{0}$ are the displacement fields determined by the nodal translational displacements and $\mathbf{u}^{\theta}$ are the additional displacement fields only determined by the vertex rigid rotations.

The element nodal displacement vector $\mathbf{q}^{e}$ is defined by

$$
\mathbf{q}^{e}=\left[\begin{array}{llllllllllll}
u_{1} & v_{1} & \theta_{1} & u_{2} & v_{2} & \theta_{2} & u_{3} & v_{3} & \theta_{3} & u_{4} & v_{4} & \theta_{4}
\end{array}\right]^{\mathrm{T}} \text {. }
$$

\section{Formulations of the New Elements QAC4 $\theta$ and QAC4 $\theta M$}

According to the definition of drilling DOFs, the element boundary displacement can be assumed as

$$
\begin{gathered}
\bar{u}_{i j}=\bar{u}_{i j}^{0}+\bar{u}_{\theta i j} \\
\bar{v}_{i j}=\bar{v}_{i j}^{0}+\bar{v}_{\theta i j}, \\
(i j=12,23,34,41),
\end{gathered}
$$

where the translational displacements $\bar{u}_{i j}$ and $\bar{v}_{i j}$ can be interpolated by the nodal displacements

$$
\begin{aligned}
& \left\{\begin{array}{c}
\bar{u}_{12}^{0} \\
\bar{v}_{12}^{0}
\end{array}\right\}=-\frac{T_{1}}{g_{1}}\left\{\begin{array}{l}
u_{1} \\
v_{1}
\end{array}\right\}-\frac{T_{2}}{g_{2}}\left\{\begin{array}{l}
u_{2} \\
v_{2}
\end{array}\right\}, \\
& \left\{\begin{array}{c}
\bar{u}_{23}^{0} \\
\bar{v}_{23}^{0}
\end{array}\right\}=-\frac{T_{2}}{g_{2}}\left\{\begin{array}{l}
u_{2} \\
v_{2}
\end{array}\right\}+\frac{T_{1}}{g_{3}}\left\{\begin{array}{l}
u_{3} \\
v_{3}
\end{array}\right\},
\end{aligned}
$$




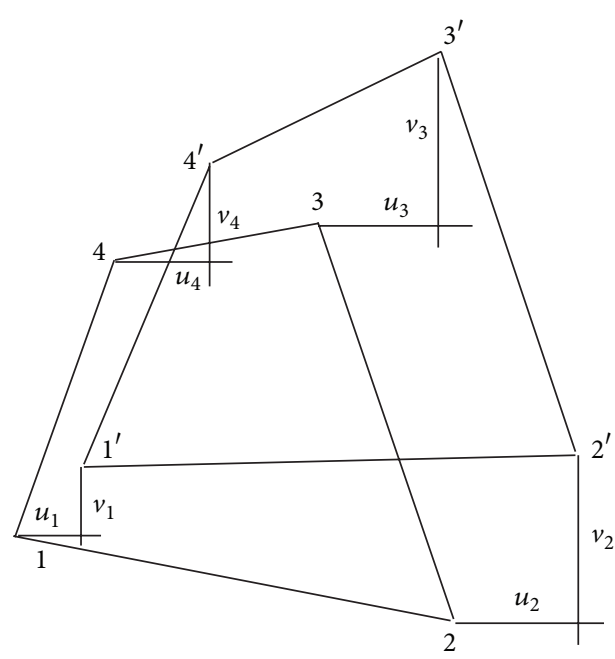

(a) Translational freedom

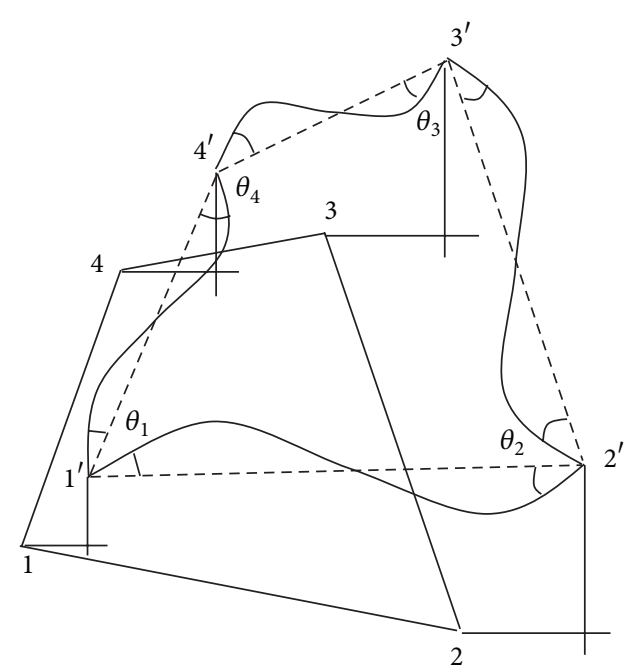

(b) Drilling freedom

Figure 5: DOFs of a membrane element.

$$
\begin{aligned}
& \left\{\begin{array}{c}
\bar{u}_{34}^{0} \\
\bar{v}_{34}^{0}
\end{array}\right\}=\frac{T_{1}}{g_{3}}\left\{\begin{array}{l}
u_{3} \\
v_{3}
\end{array}\right\}+\frac{T_{2}}{g_{4}}\left\{\begin{array}{l}
u_{4} \\
v_{4}
\end{array}\right\}, \\
& \left\{\begin{array}{l}
\bar{u}_{41}^{0} \\
\bar{v}_{41}^{0}
\end{array}\right\}=\frac{T_{2}}{g_{4}}\left\{\begin{array}{l}
u_{4} \\
v_{4}
\end{array}\right\}-\frac{T_{1}}{g_{1}}\left\{\begin{array}{l}
u_{1} \\
v_{1}
\end{array}\right\} .
\end{aligned}
$$

$$
\begin{aligned}
& \left.\frac{\partial}{\partial n}\left\{\begin{array}{c}
u_{i j}^{\theta} \\
v_{i j}^{\theta}
\end{array}\right\}\right|_{j}=\frac{1}{d_{m}}\left\{\begin{array}{c}
b_{m} \\
c_{m}
\end{array}\right\} \theta_{j}, \\
& (i j m=124,231,342,413) .
\end{aligned}
$$

The element boundary displacements caused by the additional vertex rigid rotations can be assumed by using the QACM-III:

$$
\begin{aligned}
& \left\{\begin{array}{c}
\bar{u}_{23}^{\theta} \\
\bar{v}_{23}^{\theta}
\end{array}\right\}=\left\{\begin{array}{l}
b_{1} \\
c_{1}
\end{array}\right\} T_{1} T_{2}\left(\frac{T_{2} \theta_{2}}{g_{2}^{2} g_{3}}+\frac{T_{1} \theta_{3}}{g_{2} g_{3}^{2}}\right), \\
& \left\{\begin{array}{c}
\bar{u}_{34}^{\theta} \\
\bar{v}_{34}^{\theta}
\end{array}\right\}=\left\{\begin{array}{l}
b_{2} \\
c_{2}
\end{array}\right\} T_{1} T_{2}\left(\frac{T_{1} \theta_{3}}{g_{3}^{2} g_{4}}-\frac{T_{2} \theta_{4}}{g_{3} g_{4}^{2}}\right), \\
& \left\{\begin{array}{l}
\bar{u}_{41}^{\theta} \\
\bar{v}_{41}^{\theta}
\end{array}\right\}=\left\{\begin{array}{l}
b_{3} \\
c_{3}
\end{array}\right\} T_{1} T_{2}\left(-\frac{T_{2} \theta_{4}}{g_{4}^{2} g_{1}}-\frac{T_{1} \theta_{1}}{g_{4} g_{1}^{2}}\right), \\
& \left\{\begin{array}{l}
\bar{u}_{12}^{\theta} \\
\bar{v}_{12}^{\theta}
\end{array}\right\}=\left\{\begin{array}{l}
b_{4} \\
c_{4}
\end{array}\right\} T_{1} T_{2}\left(-\frac{T_{1} \theta_{1}}{g_{1}^{2} g_{2}}+\frac{T_{2} \theta_{2}}{g_{1} g_{2}^{2}}\right) .
\end{aligned}
$$

It can be seen that, at the element corners (nodes), these boundary additional displacements are always equal to zero, and their normal derivatives of each edge are given by

$$
\left.\frac{\partial}{\partial n}\left\{\begin{array}{c}
u_{i j}^{\theta} \\
v_{i j}^{\theta}
\end{array}\right\}\right|_{i}=\frac{1}{d_{m}}\left\{\begin{array}{l}
b_{m} \\
c_{m}
\end{array}\right\} \theta_{i},
$$

The element displacement fields can be assumed in QACM-III as follows:

$$
\begin{aligned}
& u=\alpha_{1}+\alpha_{2} T_{1}+\alpha_{3} T_{2}+\alpha_{4} T_{1} T_{2}+\alpha_{5} T_{1}^{2}+\alpha_{6} T_{2}^{2} \\
& v=\beta_{1}+\beta_{2} T_{1}+\beta_{3} T_{2}+\beta_{4} T_{1} T_{2}+\beta_{5} T_{1}^{2}+\beta_{6} T_{2}^{2}
\end{aligned}
$$

In order to determine the constant $\alpha_{i}$, six generalized conforming conditions are introduced:

$$
\begin{gathered}
\sum_{i=1}^{4}(u-\bar{u})_{i}=0, \\
\sum_{i=1}^{4} \xi_{i} \eta_{i}(u-\bar{u})_{i}=0, \\
\int_{l_{i j}}(u-\bar{u}) d \bar{s}=0 \quad(i j=23,34,41,12) .
\end{gathered}
$$

The conforming conditions for $v$ are similar to those for $u$. Then, the constants $\alpha_{i}$ and $\beta_{i}$ in (21) can be solved:

$$
\begin{aligned}
& \boldsymbol{\alpha}=\mathbf{L}^{-1} \mathbf{R}_{u} \mathbf{q}_{u} \\
& \boldsymbol{\beta}=\mathbf{L}^{-1} \mathbf{R}_{v} \mathbf{q}_{v},
\end{aligned}
$$


where

$$
\begin{aligned}
& \mathbf{q}_{u}=\left[\begin{array}{llllllll}
u_{1} & u_{2} & u_{3} & u_{4} & \theta_{1} & \theta_{2} & \theta_{3} & \theta_{4}
\end{array}\right]^{\mathrm{T}} \\
& \mathbf{q}_{v}=\left[\begin{array}{llllllll}
v_{1} & v_{2} & v_{3} & v_{4} & \theta_{1} & \theta_{2} & \theta_{3} & \theta_{4}
\end{array}\right]^{\mathrm{T}}, \\
& \mathbf{L}=\left[\begin{array}{cccccc}
4 & g_{3}-g_{1} & g_{4}-g_{2} & 0 & g_{1}^{2}+g_{3}^{2} & g_{2}^{2}+g_{4}^{2} \\
0 & g_{3}-g_{1} & g_{2}-g_{4} & 0 & g_{1}^{2}+g_{3}^{2} & -g_{2}^{2}-g_{4}^{2} \\
1 & \frac{g_{3}}{2} & -\frac{g_{2}}{2} & -\frac{g_{2} g_{3}}{6} & \frac{g_{3}^{2}}{3} & \frac{g_{2}^{2}}{3} \\
1 & \frac{g_{3}}{2} & \frac{g_{4}}{2} & \frac{g_{3} g_{4}}{6} & \frac{g_{3}^{2}}{3} & \frac{g_{4}^{2}}{3} \\
1 & -\frac{g_{1}}{2} & \frac{g_{4}}{2} & -\frac{g_{4} g_{1}}{6} & \frac{g_{1}^{2}}{3} & \frac{g_{4}^{2}}{3} \\
1 & -\frac{g_{1}}{2} & -\frac{g_{2}}{2} & \frac{g_{1} g_{2}}{6} & \frac{g_{1}^{2}}{3} & \frac{g_{2}^{2}}{3}
\end{array}\right] \text {, } \\
& \mathbf{R}_{u}=\left[\begin{array}{cccccccc}
1 & 1 & 1 & 1 & 0 & 0 & 0 & 0 \\
1 & -1 & 1 & -1 & 0 & 0 & 0 & 0 \\
0 & \frac{1}{2} & \frac{1}{2} & 0 & 0 & \frac{b_{1}}{12} & -\frac{b_{1}}{12} & 0 \\
0 & 0 & \frac{1}{2} & \frac{1}{2} & 0 & 0 & \frac{b_{2}}{12} & -\frac{b_{2}}{12} \\
\frac{1}{2} & 0 & 0 & \frac{1}{2} & -\frac{b_{3}}{12} & 0 & 0 & \frac{b_{3}}{12} \\
\frac{1}{2} & \frac{1}{2} & 0 & 0 & \frac{b_{4}}{12} & -\frac{b_{4}}{12} & 0 & 0
\end{array}\right], \\
& \mathbf{R}_{v}=\left[\begin{array}{cccccccc}
1 & 1 & 1 & 1 & 0 & 0 & 0 & 0 \\
1 & -1 & 1 & -1 & 0 & 0 & 0 & 0 \\
0 & \frac{1}{2} & \frac{1}{2} & 0 & 0 & \frac{c_{1}}{12} & -\frac{c_{1}}{12} & 0 \\
0 & 0 & \frac{1}{2} & \frac{1}{2} & 0 & 0 & \frac{c_{2}}{12} & -\frac{c_{2}}{12} \\
\frac{1}{2} & 0 & 0 & \frac{1}{2} & -\frac{c_{3}}{12} & 0 & 0 & \frac{c_{3}}{12} \\
\frac{1}{2} & \frac{1}{2} & 0 & 0 & \frac{c_{4}}{12} & -\frac{c_{4}}{12} & 0 & 0
\end{array}\right] .
\end{aligned}
$$

Let

$$
\mathbf{H}_{u}=\mathbf{L}^{-1} \mathbf{R}_{u}, \quad \mathbf{H}_{v}=\mathbf{L}^{-1} \mathbf{R}_{v}
$$

Thus, the displacement fields can be written in the following:

$$
u=\sum_{i=1}^{4} N_{i}^{0} u_{i}+\sum_{i=1}^{4} N_{u \theta i} \theta_{i}, \quad v=\sum_{i=1}^{4} N_{i}^{0} v_{i}+\sum_{i=1}^{4} N_{v \theta i} \theta_{i},
$$

where the shape functions of translational displacements are

$$
\begin{aligned}
N_{i}^{0}= & H_{u, 1 i}+H_{u, 2 i} T_{1}+H_{u, 3 i} T_{2}+H_{u, 4 i} T_{1} T_{2} \\
& +H_{u, 5 i} T_{1}^{2}+H_{u, 6 i} T_{2}^{2} \\
= & H_{v, 1 i}+H_{v, 2 i} T_{1}+H_{v, 3 i} T_{2}+H_{v, 4 i} T_{1} T_{2} \\
& +H_{v, 5 i} T_{1}^{2}+H_{v, 6 i} T_{2}^{2}, \\
& (i=1,2,3,4) .
\end{aligned}
$$

And the shape functions of additional displacement fields related to the vertex rigid rotations are

$$
\begin{aligned}
N_{u \theta i}= & H_{u, 1 j}+H_{u, 2 j} T_{1}+H_{u, 3 j} T_{2}+H_{u, 4 j} T_{1} T_{2} \\
& +H_{u, 5 j} T_{1}^{2}+H_{u, 6 j} T_{2}^{2}, \\
N_{v \theta i}= & H_{v, 1 j}+H_{v, 2 j} T_{1}+H_{v, 3 j} T_{2}+H_{v, 4 j} T_{1} T_{2} \\
& +H_{v, 5 j} T_{1}^{2}+H_{v, 6 j} T_{2}^{2}, \\
& i=1,2,3,4, \quad j=i+4, \quad j>4 .
\end{aligned}
$$

The element strain fields are given by

$$
\boldsymbol{\varepsilon}=\mathbf{B}_{q} \mathbf{q}^{e}
$$

where

$$
\begin{aligned}
& \mathbf{B}_{q}=\left[\begin{array}{llll}
\mathbf{B}_{1} & \mathbf{B}_{2} & \mathbf{B}_{3} & \mathbf{B}_{4}
\end{array}\right], \\
& \mathbf{B}_{i}=\left[\begin{array}{ccc}
\frac{\partial N_{i}^{0}}{\partial x} & 0 & \frac{\partial N_{u \theta i}}{\partial x} \\
0 & \frac{\partial N_{i}^{0}}{\partial y} & \frac{\partial N_{v \theta i}}{\partial y} \\
\frac{\partial N_{i}^{0}}{\partial y} & \frac{\partial N_{i}^{0}}{\partial x} & \frac{\partial N_{u \theta i}}{\partial y}+\frac{\partial N_{v \theta i}}{\partial x}
\end{array}\right], \quad(i=1,2,3,4), \\
& \left\{\begin{array}{c}
\frac{\partial N_{i}^{0}}{\partial x} \\
\frac{\partial N_{i}^{0}}{\partial y}
\end{array}\right\}=\frac{H_{u, 2 i}}{2 A}\left\{\begin{array}{l}
y_{4}-y_{2} \\
x_{2}-x_{4}
\end{array}\right\}+\frac{H_{u, 3 i}}{2 A}\left\{\begin{array}{l}
y_{1}-y_{3} \\
x_{3}-x_{1}
\end{array}\right\} \\
& +\frac{H_{u, 4 i}}{2 A}\left\{\begin{array}{l}
\left(y_{4}-y_{2}\right) T_{2}+\left(y_{1}-y_{3}\right) T_{1} \\
\left(x_{2}-x_{4}\right) T_{2}+\left(x_{3}-x_{1}\right) T_{1}
\end{array}\right\} \\
& +\frac{H_{u, 5 i}}{A}\left\{\begin{array}{l}
\left(y_{4}-y_{2}\right) T_{1} \\
\left(x_{2}-x_{4}\right) T_{1}
\end{array}\right\} \\
& +\frac{H_{u, 6 i}}{A}\left\{\begin{array}{l}
\left(y_{1}-y_{3}\right) T_{2} \\
\left(x_{3}-x_{1}\right) T_{2}
\end{array}\right\} \text {, }
\end{aligned}
$$




$$
\begin{aligned}
\left\{\begin{array}{c}
\frac{\partial N_{u \theta i}^{0}}{\partial x} \\
\frac{\partial N_{u \theta i}^{0}}{\partial y}
\end{array}\right\}= & \frac{H_{u, 2 j}}{2 A}\left\{\begin{array}{l}
y_{4}-y_{2} \\
x_{2}-x_{4}
\end{array}\right\}+\frac{H_{u, 3 j}}{2 A}\left\{\begin{array}{l}
y_{1}-y_{3} \\
x_{3}-x_{1}
\end{array}\right\} \\
& +\frac{H_{u, 4 j}}{2 A}\left\{\begin{array}{l}
\left(y_{4}-y_{2}\right) T_{2}+\left(y_{1}-y_{3}\right) T_{1} \\
\left(x_{2}-x_{4}\right) T_{2}+\left(x_{3}-x_{1}\right) T_{1}
\end{array}\right\} \\
& +\frac{H_{u, 5 j}}{A}\left\{\begin{array}{l}
\left(y_{4}-y_{2}\right) T_{1} \\
\left(x_{2}-x_{4}\right) T_{1}
\end{array}\right\} \\
& +\frac{H_{u, 6 j}}{A}\left\{\begin{array}{l}
\left(y_{1}-y_{3}\right) T_{2} \\
\left(x_{3}-x_{1}\right) T_{2}
\end{array}\right\},
\end{aligned}
$$

and $\partial N_{v \theta i}^{0} / \partial x$ and $\partial N_{v \theta i}^{0} / \partial y$ can be obtained following a similar procedure.

Finally, the element stiffness matrix is given by

$$
\mathbf{k}_{q q}=\iint_{A} \mathbf{B}_{q}^{\mathrm{T}} \mathbf{D} \mathbf{B}_{q} t d A
$$

where $\mathbf{D}$ is the elastic matrix. This element is named QAC4 $\theta$.

In order to make further improvement on element QAC4 $\theta$, a generalized bubble displacement field $\mathbf{u}_{\lambda}$ is introduced with the following generalized conforming conditions:

$$
\int_{l_{i j}} \mathbf{u}_{\lambda} d s=\int_{l_{i j}}\left\{\begin{array}{l}
u_{\lambda} \\
v_{\lambda}
\end{array}\right\} d s=\mathbf{0}, \quad(i j=12,23,34,41) .
$$

$\mathbf{u}_{\lambda}$ is assumed to be expressed in terms of the QACM-II:

$$
\begin{aligned}
& u_{\lambda}=\lambda_{1} Z_{1}^{2}+\lambda_{2} Z_{2}^{2}+\lambda_{3} Z_{1}+\lambda_{4} Z_{2}+\lambda_{5} \\
& v_{\lambda}=\lambda_{1}^{\prime} Z_{1}^{2}+\lambda_{2}^{\prime} Z_{2}^{2}+\lambda_{3}^{\prime} Z_{1}+\lambda_{4}^{\prime} Z_{2}+\lambda_{5}^{\prime} .
\end{aligned}
$$

Substitution of (33) into (32) yields

$$
\begin{gathered}
\lambda_{2}=\lambda_{1} \\
\lambda_{3}=\frac{2\left(g_{1}-g_{2}\right)}{3} \lambda_{1} \\
\lambda_{4}=\frac{2\left(g_{2}-g_{3}\right)}{3} \lambda_{1} \\
\lambda_{5}=\frac{2\left(g_{1} g_{3}+g_{2} g_{4}\right)-5}{3} \lambda_{1} .
\end{gathered}
$$

And $\lambda_{i}^{\prime}(i=1 \sim 5)$ have similar relations. Thus, the shape functions of this additional displacement field can be obtained:

$$
\begin{aligned}
N_{\lambda 1}= & Z_{1}^{2}+Z_{2}^{2}+\frac{2\left(g_{1}-g_{2}\right)}{3} Z_{1}+\frac{2\left(g_{2}-g_{3}\right)}{3} Z_{2} \\
& +\frac{2\left(g_{1} g_{3}+g_{2} g_{4}\right)-5}{3} .
\end{aligned}
$$

Then, the corresponding strain vector can be written as

$$
\boldsymbol{\varepsilon}_{\lambda}=\left\{\begin{array}{l}
\varepsilon_{\lambda x} \\
\varepsilon_{\lambda y}
\end{array}\right\}=\mathbf{B}_{\lambda}\left\{\begin{array}{l}
\lambda_{1} \\
\lambda_{1}^{\prime}
\end{array}\right\}=\mathbf{B}_{\lambda} \lambda
$$

where

$$
\mathbf{B}_{\lambda}=\left[\begin{array}{cc}
\frac{\partial N_{\lambda 1}}{\partial x} & 0 \\
0 & \frac{\partial N_{\lambda 1}}{\partial y} \\
\frac{\partial N_{\lambda 1}}{\partial y} & \frac{\partial N_{\lambda 1}}{\partial x}
\end{array}\right],
$$

$$
\begin{aligned}
\frac{\partial N_{\lambda 1}}{\partial x}= & \frac{2\left(b_{3}-b_{1}\right)}{A} Z_{1}+\frac{2\left(b_{4}-b_{2}\right)}{A} Z_{2} \\
& +\frac{2\left(b_{3}-b_{1}\right)\left(g_{1}-g_{2}\right)}{3 A}
\end{aligned}
$$

$$
\begin{aligned}
& +\frac{2\left(b_{4}-b_{2}\right)\left(g_{2}-g_{3}\right)}{3 A} \\
\frac{\partial N_{\lambda 1}}{\partial y}= & \frac{2\left(c_{3}-c_{1}\right)}{A} Z_{1}+\frac{2\left(c_{4}-c_{2}\right)}{A} Z_{2} \\
& +\frac{2\left(c_{3}-c_{1}\right)\left(g_{1}-g_{2}\right)}{3 A} \\
& +\frac{2\left(c_{4}-c_{2}\right)\left(g_{2}-g_{3}\right)}{3 A} .
\end{aligned}
$$

The final element stiffness matrix of the element is

$$
\mathbf{k}^{e}=\mathbf{k}_{q q}-\mathbf{k}_{\lambda q}^{\mathrm{T}} \mathbf{k}_{\lambda \lambda}^{-1} \mathbf{k}_{\lambda q}
$$

where

$$
\begin{aligned}
\mathbf{k}_{q q} & =\iint \mathbf{B}_{q}^{\mathrm{T}} \mathbf{D} \mathbf{B}_{q} t d A \\
\mathbf{k}_{\lambda \lambda} & =\iint \mathbf{B}_{\lambda}^{\mathrm{T}} \mathbf{D} \mathbf{B}_{\lambda} t d A \\
\mathbf{k}_{\lambda q} & =\iint \mathbf{B}_{\lambda}^{\mathrm{T}} \mathbf{D} \mathbf{B}_{q} t d A .
\end{aligned}
$$

This element is named QAC4OM.

\section{Numerical Examples}

Seven benchmark problems, which are listed in Table 1, have been used for evaluating the performance of the elements. The results solved by the other 14 element models listed in Table 2 are also given for comparison.

Example 1 (patch test). The constant strain/stress patch test using irregular mesh is shown in Figure 6. Let Young's modulus $E=1000$, Poisson's ratio $\mu=0.25$, and thickness of the patch $t=1$. Both QAC $4 \theta$ and QAC $4 \theta \mathrm{M}$ can present exact solutions. 


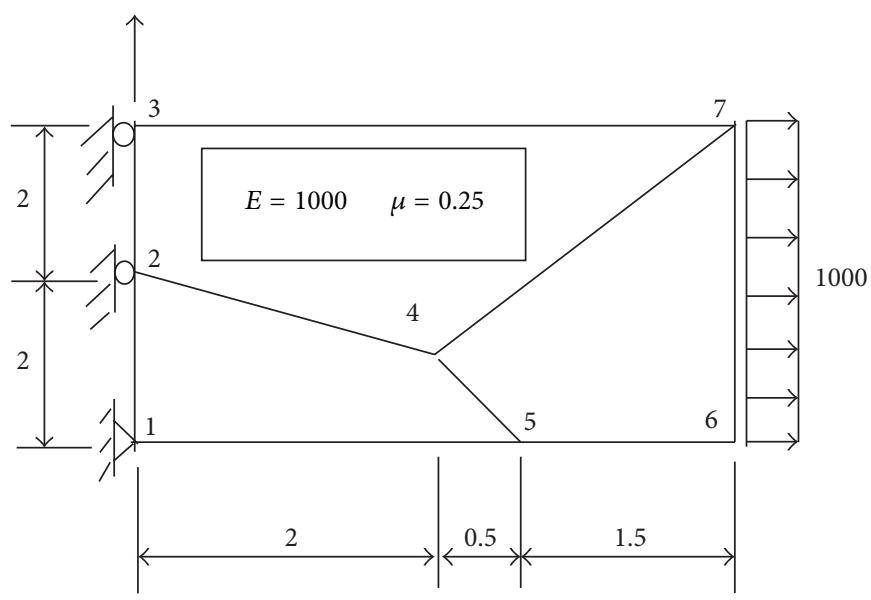

FIGURE 6: Patch test of constant stress/strain state.

TABLE 1: List of benchmark problems.

\begin{tabular}{llll}
\hline Number & \multicolumn{1}{c}{ Benchmark problems (figure number) } & & \\
\hline 1 & Patch test & (Figure 6) & \\
2 & Cooks skew beam & (Figure 7) & Table 3 \\
3 & Beam divided by five quadrilateral elements & (Figure 8) & Table 4 \\
4 & Beam divided by four quadrilateral elements & (Figure 9) & Table 5 \\
5 & MacNeal's thin beam & (Figure 10) & Table 6 \\
6 & Thin curving beam & (Figure 11) & Table 7 \\
7 & Beam divided by two elements with distortion parameter & (Figure 12) & Table 8 \\
\hline
\end{tabular}

TABLE 2: List of element models for comparison.

\begin{tabular}{lccc}
\hline Number & & Element model & Reference \\
\hline 1 & Q4 & 4-node isoparametric element & Wilson et al. [55] \\
2 & Q6 & 4-node isoparametric element with internal parameters & Taylor et al. [56] \\
3 & QM6 & 4-node isoparametric element with internal parameters & Pian and Sumihara [57] \\
4 & P-S & Hybrid stress element & MacNeal and Harder [32] \\
5 & QUAD4 & 4-node element in MSC/NASTRAN & MacNeal and Harder [8] \\
6 & Q4S & Membrane element with drilling DOFs & Long and Xu [23] \\
7 & GQ12 & Membrane element with drilling DOFs & Long and Xu [23] \\
8 & GQ12M8 & Membrane element with drilling DOFs & Ibrahimbegovic et al. [11] \\
10 & D-type & Membrane element with drilling DOFs & Groenwold and Stander [58] \\
11 & Groenwold1995 & Membrane element with drilling DOFs & Aminpour [59] \\
12 & AQR8 & Membrane element with drilling DOFs & Chen and Cheung [60] \\
13 & RGD20 & Refined hybrid element & \\
14 & Q8 & 8-node isoparametric element & \\
\hline
\end{tabular}

Example 2 (Cook's skew beam). This example, in which a skew cantilever with shear distributed load at the free edge, as shown in Figure 7, was proposed by Cook et al. [61]. The results of vertical deflection at point $\mathrm{C}$, the maximum principal stress at point $\mathrm{A}$, and the minimum principal stress at point $\mathrm{B}$ are listed in Table 3.

Example 3 (cantilever beam divided by five quadrilateral elements). The cantilever beam, as shown in Figure 8, is divided by five irregular quadrilateral elements. And two loading cases are considered: (a) pure bending under moment $M$ and (b) linear bending under transverse force $P$. Young's modulus $E=1500$, and Poisson's ratio $v=0.25$. The results of the vertical deflection $v_{\mathrm{A}}$ at point $\mathrm{A}$ and the stress $\sigma_{x \mathrm{~B}}$ at point $\mathrm{B}$ are given in Table 4 .

Example 4 (cantilever beam divided by five quadrilateral elements). As shown in Figure 9, the cantilever beam is 
TABLE 3: Results of Cook's beam.

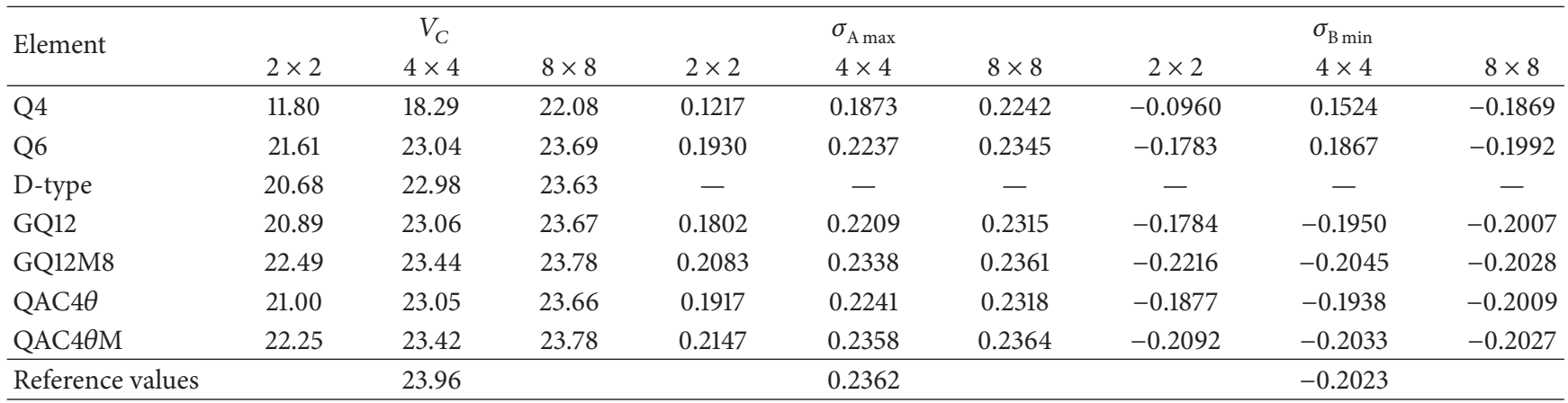

TABLE 4: Results of cantilever beam with five elements.

\begin{tabular}{lcccc}
\hline \multirow{2}{*}{ Elements } & \multicolumn{2}{c}{ Load I } & \multicolumn{2}{c}{ Load II } \\
& $v_{\mathrm{A}}$ & $\sigma_{x \mathrm{~B}}$ & $v_{\mathrm{A}}$ & $\sigma_{x \mathrm{~B}}$ \\
\hline Q4 & 45.7 & -1761 & 50.7 & -2448 \\
Q6 & 98.4 & -2428 & 100.4 & -3354 \\
GQ12 & 95.5 & -2989 & 96.0 & -4096 \\
GQ12M8 & 100.0 & -3000 & 101.0 & -4147 \\
QAC40 & 100.0 & -3000 & 98.6 & -3931 \\
QAC40M & 100.0 & -3000 & 101.0 & -3977 \\
Exact & 100.0 & -3000 & 102.6 & -4050 \\
\hline
\end{tabular}

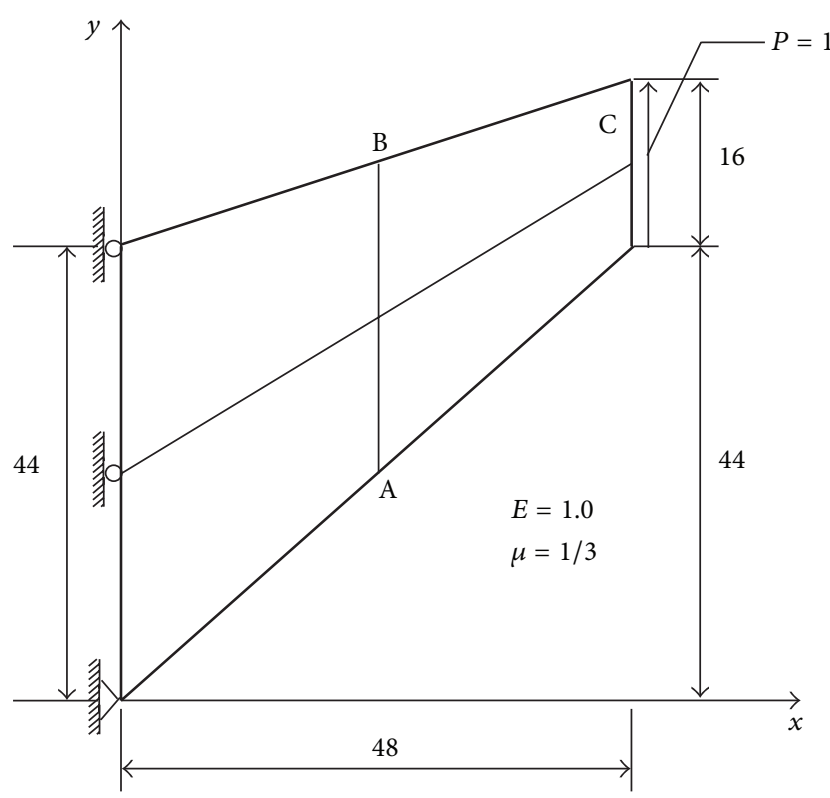

FIgURE 7: Cook's skew beam problem.

divided by four irregular quadrilateral elements. The results of the deflections at the tip points $\mathrm{A}$ and $\mathrm{B}$ are shown in Table 5.

Example 5 (MacNeal's beam). Consider the thin beams presented in Figure 10. Three different mesh shapes, rectangular, parallelogram, and trapezoidal, are adopted. This example, proposed by MacNeal and Harder [32], is a famous

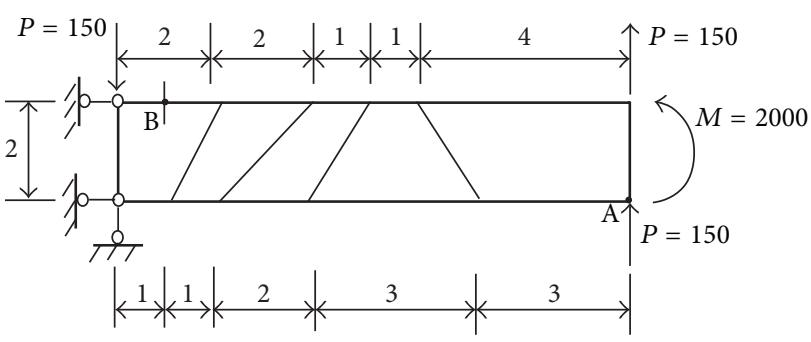

FIgURE 8: Cantilever beam with five irregular elements.

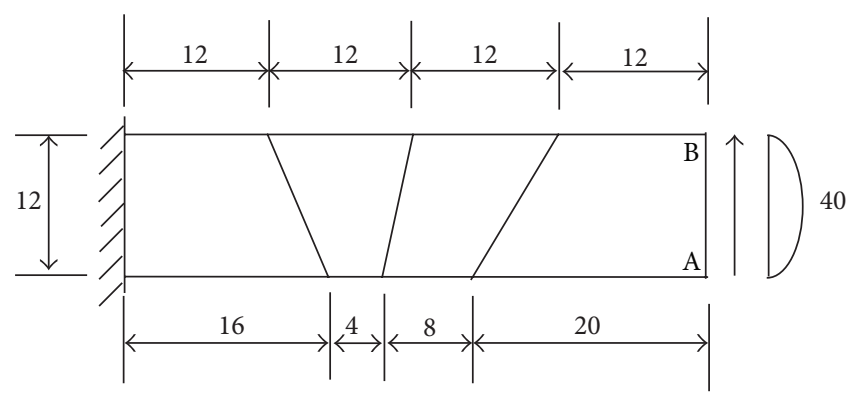

FIGURE 9: Cantilever beam modeled with four irregular elements.

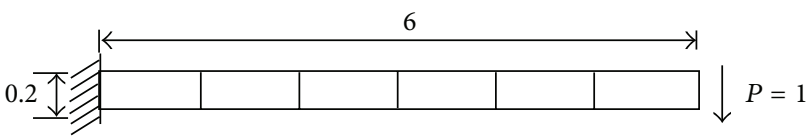

(a)

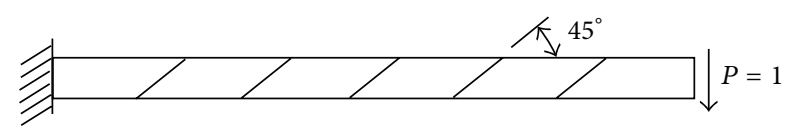

(b)

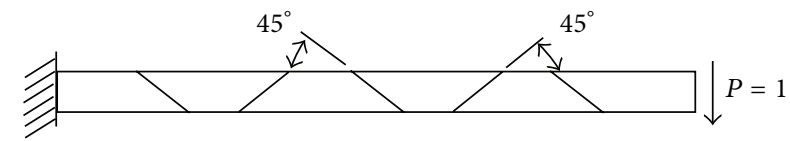

(c)

Figure 10: MacNeal's beam. 
TABLE 5: Results of cantilever with four elements.

\begin{tabular}{|c|c|c|c|c|c|c|}
\hline \multirow{2}{*}{ Elements } & \multicolumn{3}{|c|}{ Tip deflections } & \multicolumn{3}{|c|}{ Normalized values } \\
\hline & Point A & Point B & Average & Point A & Point B & Average \\
\hline D-type & - & - & 0.3065 & - & - & 0.861 \\
\hline $\mathrm{Q} 4 \mathrm{~S}$ & - & - & 0.2978 & - & - & 0.837 \\
\hline Groenwold1995 & - & - & 0.3086 & - & - & 0.867 \\
\hline GQ12 & 0.3337 & 0.3324 & 0.3331 & 0.938 & 0.934 & 0.936 \\
\hline GQ12M & 0.3420 & 0.3404 & 0.3412 & 0.961 & 0.957 & 0.959 \\
\hline $\mathrm{QAC} 4 \theta$ & 0.3523 & 0.3516 & 0.3520 & 0.990 & 0.988 & 0.989 \\
\hline $\mathrm{QAC} 4 \theta \mathrm{M}$ & 0.3523 & 0.3516 & 0.3520 & 0.990 & 0.988 & 0.989 \\
\hline Reference value & \multicolumn{3}{|c|}{0.3558} & \multicolumn{3}{|c|}{1.000} \\
\hline
\end{tabular}

TABLE 6: The normalized results of the tip deflection for MacNeal's beam.

\begin{tabular}{|c|c|c|c|c|c|c|}
\hline \multirow{2}{*}{ Elements } & \multicolumn{3}{|c|}{ Load $P$} & \multicolumn{3}{|c|}{ Load $M$} \\
\hline & Mesh (a) & Mesh (b) & Mesh (c) & $\operatorname{Mesh}(\mathrm{a})$ & Mesh (b) & Mesh (c) \\
\hline Q4 & 0.093 & 0.035 & 0.003 & 0.093 & 0.031 & 0.022 \\
\hline Q6 & 0.993 & 0.677 & 0.106 & 1.000 & 0.759 & 0.093 \\
\hline QM6 & 0.993 & 0.623 & 0.044 & 1.000 & 0.722 & 0.037 \\
\hline QUAD4 & 0.904 & 0.080 & 0.071 & - & - & - \\
\hline P-S & 0.993 & 0.798 & 0.221 & 1.000 & 0.852 & 0.167 \\
\hline RGD20 & 0.981 & 0.625 & 0.047 & - & - & - \\
\hline AQR8 & 0.993 & 0.986 & 0.977 & - & - & - \\
\hline Q4S & 0.993 & 0.986 & 0.988 & - & - & - \\
\hline QAC4 $\theta$ & 0.904 & 0.867 & 0.906 & 0.910 & 0.8804 & 0.930 \\
\hline $\mathrm{QAC} 4 \theta \mathrm{M}$ & 0.993 & 0.984 & 0.988 & 1.000 & 0.992 & 0.998 \\
\hline Exact & \multicolumn{3}{|c|}{$1.000(-0.1081)$} & \multicolumn{3}{|c|}{$1.000(-0.0054)$} \\
\hline
\end{tabular}

TABLE 7: The tip deflection of a thin curving beam.

\begin{tabular}{lccccccrr}
\hline $\mathrm{h} / \mathrm{R}$ & $\mathrm{Q} 4$ & QM6 & QUAD4 & GQ12 & GQ12M & QAC40 & QAC40M & Exact \\
\hline 0.03 & 0.024 & 0.339 & 0.615 & 0.670 & 0.897 & 0.712 & 1.000 & 1.000 \\
0.006 & 0.001 & 0.022 & 0.163 & 0.612 & 0.896 & 0.645 & 1.008 & 1.000 \\
\hline
\end{tabular}

benchmark for testing the sensitivity to mesh distortion of the 4-node quadrilateral membrane elements.

There are two loading cases under consideration: pure bending and transverse linear bending. Young's modulus of the beam $E=10^{7}$; Poisson's ratio $\mu=0.3$; the thickness of the beam $t=0.1$. The results of the tip deflection are shown in Table 6.

Example 6 (thin curving beam). As shown in Figure 11, a cantilever thin curving beam is subjected to a transverse force at the tip. And it is also divided by five elements. Two thickness-radius ratios, (i) $h / R=0.03$ and (ii) $h / R=0.006$, are considered. The results of the tip displacement are listed in Table 7.

Example 7 (cantilever beam divided by two elements containing a parameter of distortion). The cantilever beam shown in Figure 12 is divided by two elements. The shape of the two elements varies with the distorted parameter $e$. When
TABLE 8: Results of the tip deflection of a cantilever beam with distorted parameter $e$.

\begin{tabular}{|c|c|c|c|c|c|c|c|}
\hline$e$ & 0 & 0.5 & 1 & 2 & 3 & 4 & 4.9 \\
\hline Q4 & 28.0 & 21.0 & 14.1 & 9.7 & 8.3 & 7.2 & 6.2 \\
\hline Q8 & 100 & 100 & 99.3 & 89.3 & 59.7 & 31.6 & 19.0 \\
\hline QM6 & 100 & 80.9 & 62.7 & 54.4 & 53.6 & 51.2 & 46.8 \\
\hline P-S & 100 & 81.0 & 62.9 & 55.0 & 54.7 & 53.1 & 49.8 \\
\hline GQ12 & 100 & 97.9 & 86.3 & 48.7 & 24.9 & 13.3 & 8.0 \\
\hline GQ12M & 100 & 98.7 & 93.9 & 74.1 & 51.0 & 33.4 & 23.3 \\
\hline QAC4 $\theta$ & 100 & 99.9 & 98.9 & 99.8 & 102.0 & 102.2 & 100.3 \\
\hline QAC $4 \theta \mathrm{M}$ & 100 & 100 & 100 & 100 & 100 & 100 & 100 \\
\hline
\end{tabular}

Exact

100

$e=0$, both elements are rectangular. But with the increase of $e$, the mesh will be distorted more and more seriously. This is another famous benchmark for testing the sensitivity to the mesh distortion. For pure bending problem, the results of the tip deflection at point $\mathrm{A}$ are listed in Table 8. 


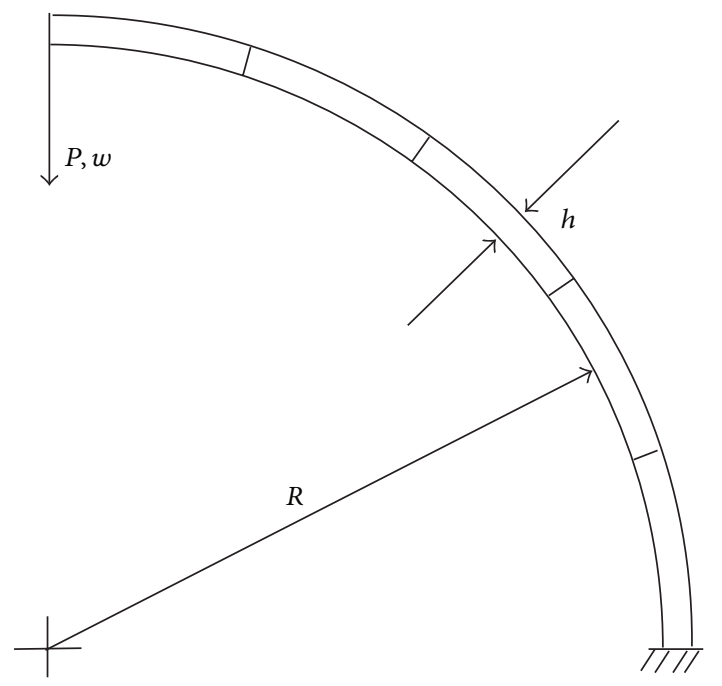

FIGURE 11: Bending of a thin curved beam.

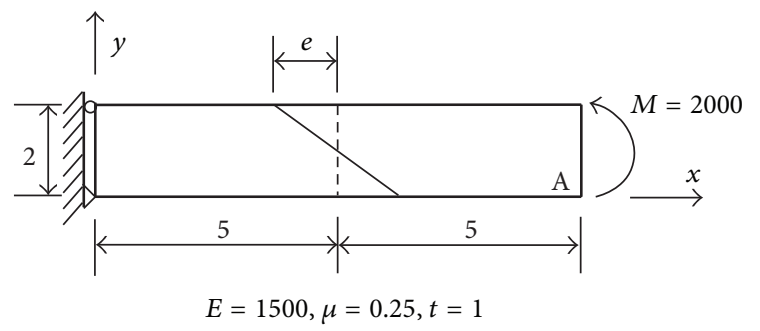

FIgURE 12: Cantilever beam divided by two elements.

\section{Conclusions}

In this paper, two membrane elements with drilling DOFs, named QAC4 $\theta$ and QAC $4 \theta \mathrm{M}$, are developed by using the quadrilateral area coordinate methods QACM-II and QACM-III. In their formulations, the additional rigid rotations at the element nodes are considered as the drilling DOFs, so that these two elements can allow the change of the angle between two adjacent sides along with the element deformations. Furthermore, since the quadrilateral area coordinates can keep the order of the Cartesian coordinates unchangeable while the mesh is distorted, the new elements exhibit better performance than other similar models and insensitivity to mesh distortion. It is demonstrated again that the quadrilateral area coordinate methods are effective tools for developing high-performance quadrilateral finite element models.

\section{Conflict of Interests}

The authors declare no conflict of interests regarding the publication of this paper.

\section{Acknowledgments}

The authors would like to acknowledge the financial support of the National Natural Science Foundation of China
(11272181), the Specialized Research Fund for the Doctoral Program of Higher Education of China (20120002110080), and the National Basic Research Program of China (Project no. 2010CB832701).

\section{References}

[1] M. D. Olson and T. W. Bearden, "A simple flat shell element revisited," International Journal for Numerical Methods in Engineering, vol. 14, no. 1, pp. 51-68, 1979.

[2] G. A. Mohr, "Finite element formulation by nested interpolations: application to the drilling freedom problem," Computers \& Structures, vol. 15, no. 2, pp. 185-190, 1982.

[3] D. J. Allman, "A compatible triangular element including vertex rotations for plane elasticity analysis," Computers and Structures, vol. 19, no. 1-2, pp. 1-8, 1984.

[4] D. J. Allman, "Evaluation of the constant strain triangle with drilling rotations," International Journal for Numerical Methods in Engineering, vol. 26, no. 12, pp. 2645-2655, 1988.

[5] P. G. Bergan and C. A. Felippa, "A triangular membrane element with rotational degrees of freedom," Computer Methods in Applied Mechanics and Engineering, vol. 50, no. 1, pp. 25-69, 1985.

[6] R. D. Cook, "A plane hybrid element with rotational D.O.F. and adjustable stiffness," International Journal for Numerical Methods in Engineering, vol. 24, no. 8, pp. 1499-1508, 1987.

[7] R. D. Cook, "Some options for plane triangular elements with rotational degrees of freedom," Finite Elements in Analysis and Design, vol. 6, no. 3, pp. 245-249, 1990.

[8] R. H. MacNeal and R. L. Harder, "A refined four-noded membrane element with rotational degrees of freedom," Computers \& Structures, vol. 28, no. 1, pp. 75-84, 1988.

[9] S. M. Yunus, S. Saigal, and R. D. Cook, "On improved hybrid finite elements with rotational degrees of freedom," International Journal for Numerical Methods in Engineering, vol. 28, no. 4, pp. 785-800, 1989.

[10] T. J. R. Hughes and F. Brezzi, "On drilling degrees of freedom," Computer Methods in Applied Mechanics and Engineering, vol. 72, no. 1, pp. 105-121, 1989.

[11] A. Ibrahimbegovic, R. L. Taylor, and E. L. Wilson, "A robust quadrilateral membrane finite element with drilling degrees of freedom," International Journal for Numerical Methods in Engineering, vol. 30, no. 3, pp. 445-457, 1990.

[12] M. Iura and S. N. Atluri, "Formulation of a membrane finite element with drilling degrees of freedom," Computational Mechanics, vol. 9, no. 6, pp. 417-428, 1992.

[13] A. Cazzani and S. N. Atluri, "Four-noded mixed finite elements, using unsymmetric stresses, for linear analysis of membranes," Computational Mechanics, vol. 11, no. 4, pp. 229-251, 1993.

[14] R. Piltner and R. L. Taylor, "Triangular finite elements with rotational degrees of freedom and enhanced strain modes," Computers and Structures, vol. 75, no. 4, pp. 361-368, 2000.

[15] S. Geyer and A. A. Groenwold, "Two hybrid stress membrane finite element families with drilling rotations," International Journal for Numerical Methods in Engineering, vol. 53, no. 3, pp. 583-601, 2002.

[16] G. Pimpinelli, "An assumed strain quadrilateral element with drilling degrees of freedom," Finite Elements in Analysis and Design, vol. 41, no. 3, pp. 267-283, 2004.

[17] A. A. Groenwold, Q. Z. Xiao, and N. J. Theron, "Accurate solution of traction free boundaries using hybrid stress membrane 
elements with drilling degrees of freedom," Computers and Structures, vol. 82, no. 23-26, pp. 2071-2081, 2004.

[18] N. Choi, Y. S. Choo, and B. C. Lee, "A hybrid Trefftz plane elasticity element with drilling degrees of freedom," Computer Methods in Applied Mechanics and Engineering, vol. 195, no. 3336, pp. 4095-4105, 2006.

[19] Y. S. Choo, N. Choi, and B. C. Lee, "Quadrilateral and triangular plane elements with rotational degrees of freedom based on the hybrid Trefftz method," Finite Elements in Analysis and Design, vol. 42, no. 11, pp. 1002-1008, 2006.

[20] H. Zhang and J. S. Kuang, "Eight-node membrane element with drilling degrees of freedom for analysis of in-plane stiffness of thick floor plates," International Journal for Numerical Methods in Engineering, vol. 76, no. 13, pp. 2117-2136, 2008.

[21] S. Kugler, P. A. Fotiu, and J. Murin, "A highly efficient membrane finite element with drilling degrees of freedom," Acta Mechanica, vol. 213, no. 3-4, pp. 323-348, 2010.

[22] S. Cen, M.-J. Zhou, and X.-R. Fu, "A 4-node hybrid stressfunction (HS-F) plane element with drilling degrees of freedom less sensitive to severe mesh distortions," Computers and Structures, vol. 89, no. 5-6, pp. 517-528, 2011.

[23] Y.-Q. Long and Y. Xu, "Generalized conforming quadrilateral membrane element with vertex rigid rotational freedom," Computers \& Structures, vol. 52, no. 4, pp. 749-755, 1994.

[24] Y. Q. Long and Y. Xu, "Generalized conforming triangular membrane element with vertex rigid rotational freedoms," Finite Elements in Analysis and Design, vol. 17, no. 4, pp. 259271, 1994.

[25] Y.-Q. Long, S. Cen, and Z.-F. Long, Advanced Finite Element Method in Structural Engineering, Springer, Berlin, Germany, 2009.

[26] Ü. H. Çalik Karaköse and H. Askes, "Static and dynamic convergence studies of a four-noded membrane finite element with rotational degrees of freedom based on displacement superposition," International Journal for Numerical Methods in Biomedical Engineering, vol. 26, no. 10, pp. 1263-1275, 2010.

[27] N.-S. Lee and K.-J. Bathe, "Effects of element distortions on the performance of isoparametric elements," International Journal for Numerical Methods in Engineering, vol. 36, no. 20, pp. 35533576, 1993.

[28] Y.-Q. Long, J.-X. Li, Z.-F. Long, and S. Cen, "Area co-ordinates used in quadrilateral elements," Communications in Numerical Methods in Engineering, vol. 15, no. 8, pp. 533-545, 1999.

[29] Z.-F. Long, J.-X. Li, S. Cen, and Y.-Q. Long, "Some basic formulae for area co-ordinates in quadrilateral elements," Communications in Numerical Methods in Engineering, vol. 15, no. 12, pp. 841-852, 1999.

[30] A.-K. Soh, Y.-Q. Long, and S. Cen, "Development of eight-node quadrilateral membrane elements using the area coordinates method," Computational Mechanics, vol. 25, no. 4, pp. 376-384, 2000.

[31] X.-M. Chen, S. Cen, Y.-Q. Long, and Z.-H. Yao, "Membrane elements insensitive to distortion using the quadrilateral area coordinate method," Computers \& Structures, vol. 82, no. 1, pp. 35-54, 2004.

[32] R. H. MacNeal and R. L. Harder, "A proposed standard set of problems to test finite element accuracy," Finite Elements in Analysis and Design, vol. 1, no. 1, pp. 3-20, 1985.

[33] S. Cen, Y. Du, X.-M. Chen, and X.-R. Fu, "The analytical element stiffness matrix of a recent 4-node membrane element formulated by the quadrilateral area co-ordinate method,"
Communications in Numerical Methods in Engineering, vol. 23, no. 12, pp. 1095-1110, 2007.

[34] S. Cen, X.-M. Chen, and X.-R. Fu, "Quadrilateral membrane element family formulated by the quadrilateral area coordinate method," Computer Methods in Applied Mechanics and Engineering, vol. 196, no. 41-44, pp. 4337-4353, 2007.

[35] Y. Du and S. Cen, "Geometrically nonlinear analysis with a 4node membrane element formulated by the quadrilateral area coordinate method," Finite Elements in Analysis and Design, vol. 44, no. 8, pp. 427-438, 2008.

[36] R. P. R. Cardoso, J. W. Yoon, and R. A. F. Valente, "A new approach to reduce membrane and transverse shear locking for one-point quadrature shell elements: linear formulation," International Journal for Numerical Methods in Engineering, vol. 66, no. 2, pp. 214-249, 2006.

[37] R. P. R. Cardoso, J. W. Yoon, and R. A. F. Valente, "Enhanced one-point quadrature shell element for nonlinear applications," International Journal for Numerical Methods in Engineering, vol. 69, no. 3, pp. 627-663, 2007.

[38] J. W. Yoon, R. P. R. Cardoso, and R. E. Dick, "Puncture fracture in an aluminum beverage can," International Journal of Impact Engineering, vol. 37, no. 2, pp. 150-160, 2010.

[39] Z. Wang and Q. Sun, "Corotational nonlinear analyses of laminated shell structures using a 4-node quadrilateral flat shell element with drilling stiffness," Acta Mechanica Sinica, vol. 30, no. 3, pp. 418-429, 2014.

[40] X.-M. Chen, S. Cen, Y.-G. Li, and J.-Y. Sun, "Several treatments on nonconforming element failed in the strict patch test," Mathematical Problems in Engineering, vol. 2013, Article ID 901495, 7 pages, 2013.

[41] G. Li, "A four-node plane parametric element based on quadrilateral area coordinate and its application to coupled soliddeformation/fluid-flow simulation for porous geomaterials," International Journal for Numerical and Analytical Methods in Geomechanics, 2014.

[42] R. P. R. Cardoso and J. W. Yoon, "One point quadrature shell elements: a study on convergence and patch tests," Computational Mechanics, vol. 40, no. 5, pp. 871-883, 2007.

[43] G. Prathap and V. Senthilkumar, "Making sense of the quadrilateral area coordinate membrane elements," Computer Methods in Applied Mechanics and Engineering, vol. 197, no. 49-50, pp. 4379-4382, 2008.

[44] R. Flajs, S. Cen, and M. Saje, "On convergence of nonconforming convex quadrilateral finite elements AGQ6," Computer Methods in Applied Mechanics and Engineering, vol. 199, no. 2528, pp. 1816-1827, 2010.

[45] A.-K. Soh, Z.-F. Long, and S. Cen, "Development of a new quadrilateral thin plate element using area coordinates," Computer Methods in Applied Mechanics and Engineering, vol. 190, no. 8-10, pp. 979-987, 2000.

[46] S. Cen, Y.-Q. Long, Z.-H. Yao, and S.-P. Chiew, "Application of the quadrilateral area co-ordinate method: a new element for Mindlin-Reissner plate," International Journal for Numerical Methods in Engineering, vol. 66, no. 1, pp. 1-45, 2006.

[47] S. Cen, X. Fu, Y. Long, H. Li, and Z. Yao, "Application of the quadrilateral area coordinate method: a new element for laminated composite plate bending problems," Acta Mechanica Sinica, vol. 23, no. 5, pp. 561-575, 2007.

[48] Y. Q. Zhu, Z. Q. Lin, and W. G. Zhang, "A quadrilateral thin shell element based on area co-ordinate for explicit dynamic analysis," Communications in Numerical Methods in Engineering, vol. 19, no. 3, pp. 169-178, 2003. 
[49] Y. Zhu and C. Wu, "An improved area-coordinate based quadrilateral shell element in dynamic explicit fea," International Journal of Modern Physics B, vol. 19, no. 1-3, pp. 687-690, 2005.

[50] M. Brunet and F. Sabourin, "Analysis of a rotation-free 4-node shell element," International Journal for Numerical Methods in Engineering, vol. 66, no. 9, pp. 1483-1510, 2006.

[51] F. Sabourin, J. Carbonniere, and M. Brunet, "A new quadrilateral shell element using 16 degrees of freedom," Engineering Computations, vol. 26, no. 5, pp. 500-540, 2009.

[52] X.-M. Chen, S. Cen, X.-R. Fu, and Y.-Q. Long, "A new quadrilateral area coodinate method (QACM-II) for developing quadrilateral finite element models," International Journal for Numerical Methods in Engineering, vol. 73, no. 13, pp. 1911-1941, 2008.

[53] S. Cen, X.-M. Chen, C.-F. Li, and X.-R. Fu, "Quadrilateral membrane elements with analytical element stiffness matrices formulated by the new quadrilateral area coordinate method (QACM-II)," International Journal for Numerical Methods in Engineering, vol. 77, no. 8, pp. 1172-1200, 2009.

[54] Z. F. Long, S. Cen, L. Wang, X. R. Fu, and Y. Q. Long, “The third form of the quadrilateral area coordinate method (QACMIII): theory, application, and scheme of composite coordinate interpolation," Finite Elements in Analysis and Design, vol. 46, no. 10, pp. 805-818, 2010.

[55] E. L. Wilson, R. L. Taylor, W. P. Doherty, and T. Ghabussi, "Incompatible displacement models," in Numerical Computational Methods in Structural Mechanics, S. T. Fenven et al., Ed., pp. 43-57, Academic Press, New York, NY, USA, 1973.

[56] R. L. Taylor, P. J. Beresford, and E. L. Wilson, "A nonconforming element for stress analysis," International Journal for Numerical Methods in Engineering, vol. 10, no. 6, pp. 12111219,1976

[57] T. H. H. Pian and K. Sumihara, "Rational approach for assumed stress finite elements," International Journal for Numerical Methods in Engineering, vol. 20, no. 9, pp. 1685-1695, 1984.

[58] A. A. Groenwold and N. Stander, "An efficient 4-node 24 D.O.F. thick shell finite element with 5-point quadrature," Engineering Computations, vol. 12, no. 8, pp. 723-747, 1995.

[59] M. A. Aminpour, "An assumed-stress hybrid 4-node shell element with drilling degrees of freedom," International Journal for Numerical Methods in Engineering, vol. 33, no. 1, pp. 19-38, 1992.

[60] W. J. Chen and Y. K. Cheung, "Three-dimensional 8-node and 20-node refined hybrid isoparametric elements," International Journal for Numerical Methods in Engineering, vol. 35, no. 9, pp. 1871-1889, 1992.

[61] R. D. Cook, D. S. Malkus, and M. E. Plesha, Concepts and Applications of Finite Element Analysis, John Wiley \& Sons, New York, NY, USA, 3rd edition, 1989. 


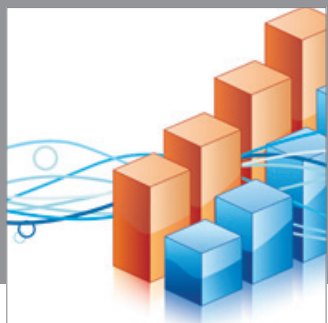

Advances in

Operations Research

mansans

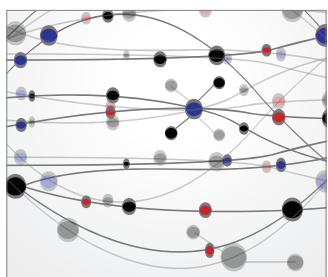

The Scientific World Journal
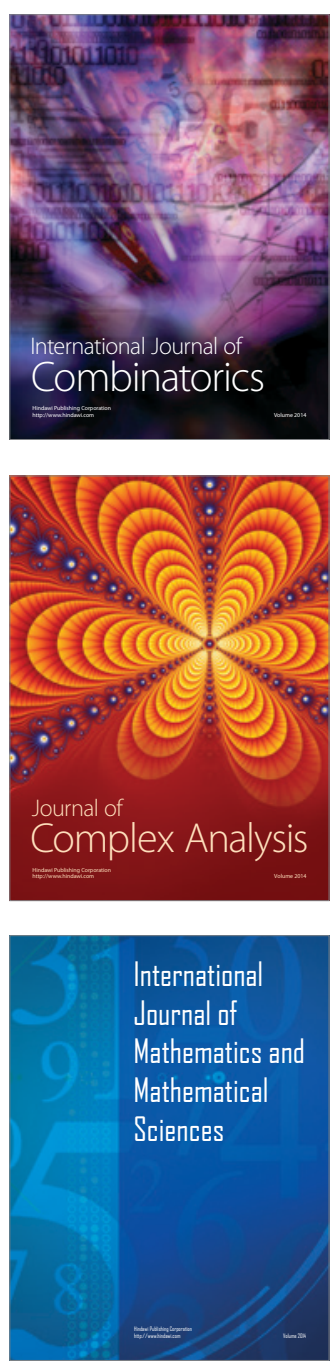
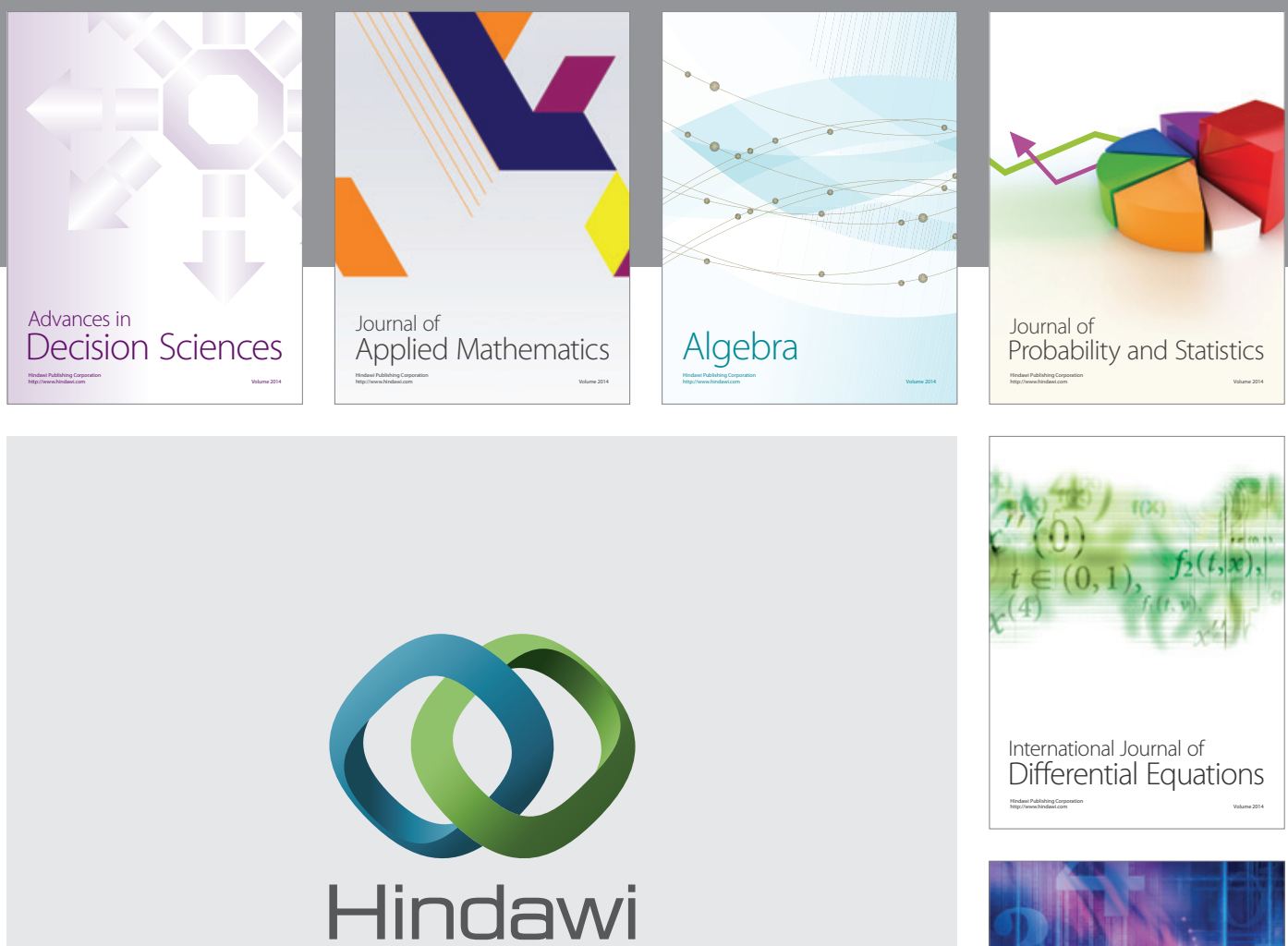

Submit your manuscripts at http://www.hindawi.com
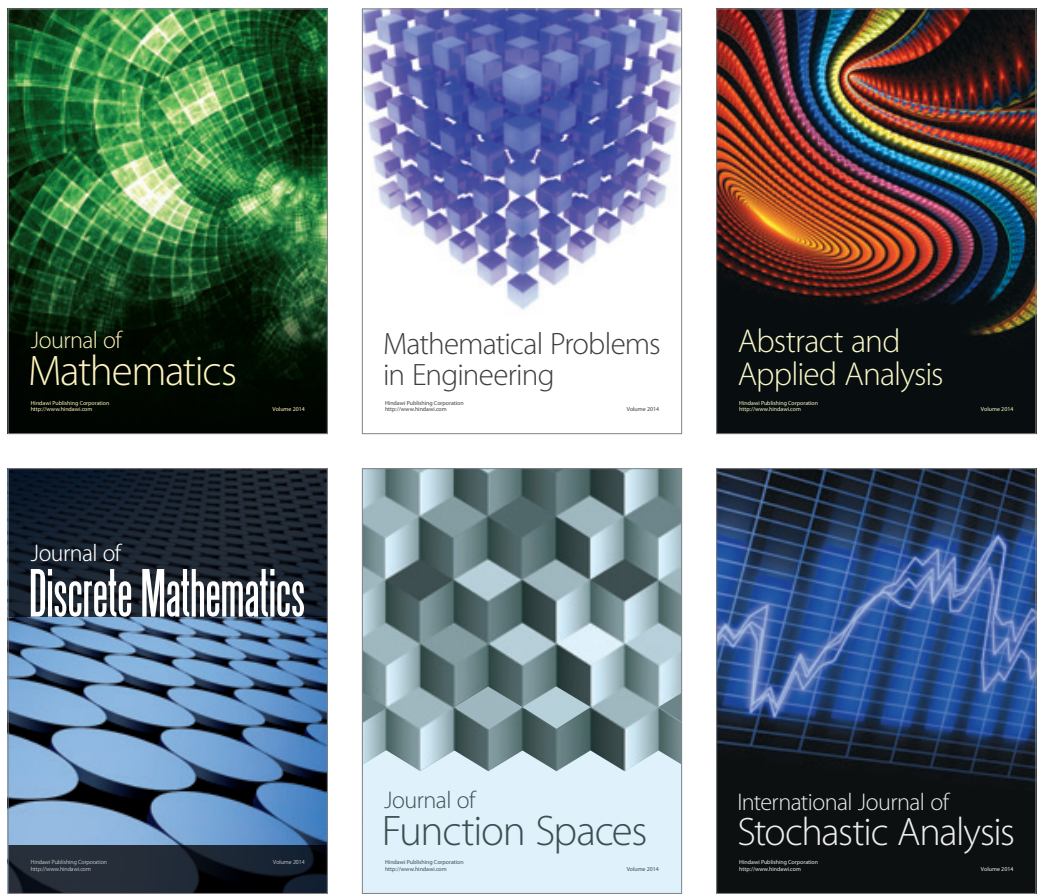

Journal of

Function Spaces

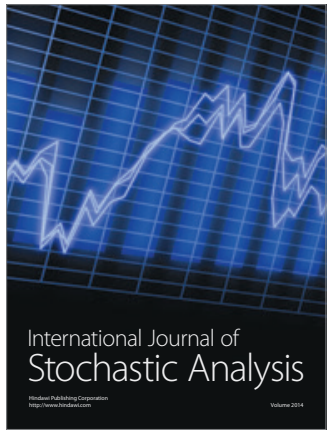

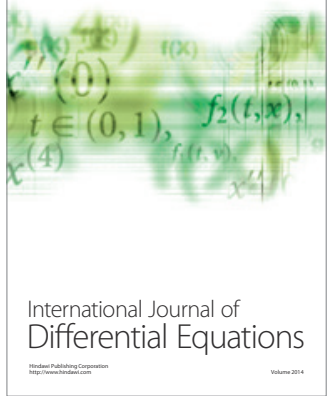
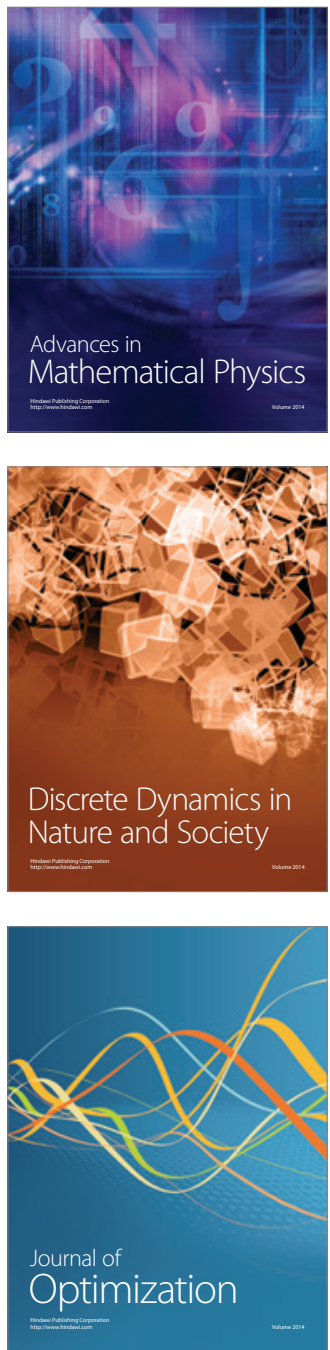